\title{
Locational Accuracy of VIP Indices for Voltage Collapse Margin Estimation
}

\author{
Miroslav Begovic \\ Texas A\&M University \\ begovic@tamu.edu
}

\author{
Aaqib Peerzada \\ Texas A\&M University \\ peerzada@tamu.edu
}

\author{
Reynaldo Nuqui \\ ABB Corporate Research \\ reynaldo.nuqui@us.abb.com
}

\author{
Ben Picone \\ ERCOT \\ bpicone@tamu.edu
}

\begin{abstract}
Nearly two decades of work on VIP (Voltage Instability Prediction) has enhanced the ability to obtain information on system vulnerability to voltage collapse based on minimum local information. Several indices have been developed over the past 20 years for local monitoring of voltage collapse problems, each with a different level of complexity with respect to computational and communicational infrastructure needed. This article addresses the disparity found in the VIP-derived margins and attempts to study the allocation of critical (more accurate) VIP locations across a power network during system changes. Additionally, a sensitivity metric is proposed to track the accurate VIP locations in real-time under increased system loading which could also lead to a meaningful data fusion of the more accurate VIPderived margins.
\end{abstract}

Keywords: power system voltage stability, VIP indicator, stability monitoring, sensor fusion.

\section{Introduction}

The phenomenon of voltage instability in power systems [1],[2],[3],[4] has received considerable attention over the past three decades. The occurrences of voltage stability are becoming more likely owing to the fact that the transmission systems are now being operated closer to their stability limits. Several blackouts that struck North America and Europe have been related to voltage instability problems. Those disturbances arise when the combined capabilities of transmission and generation are unable to meet the increasing load demand [5]. This leads to deterioration of the voltage profile in certain parts of the system, and ultimately may lead to a collapse of voltages and total system blackout.

One of the methods of analyzing voltage instability is by assessing the security margins and ensuring adequate margins against credible contingencies. For assessment of the proximity to voltage collapse, several methods based on the power flow and dynamic simulations have been used. However, these methods lack the simplicity and the computational ease found in the methods based on the local measurements. One such early method based on the local measurement of voltage and current phasors was developed in the late 90 s, commonly referred to as VIP, an acronym for Voltage Instability Prediction [6]. It proposes quasiThévenin equivalent to model the rest of the system behind a load bus. The quasi-Thévenin equivalent aggregates all other network parameters behind the point of measurement and compares them with the driving point impedance of the load. In [7], the authors expand the results to include nonlinear (ZIP) load models and propose a mechanism to monitor the generator reactive reserves in the form a real-time index. An early effort made in the direction of getting rid of some of the identification problems is compiled in [8] in which the authors propose to factor in the direction of the change of Thévenin voltage and the amount of variation in compliance with some basic rules.

An alternative method based on monitoring of the distribution voltages controlled by Load Tap Changers (LTCs) is described in [9]. The concept of networked VIP was explored in [10]. For the purpose of increasing the robustness of the VIP estimates, this method incorporates the information in the surrounding areas to further refine the estimation of Thevenin Equivalent. This technique, referred to as $\mathrm{VIP}++$, measures the voltage and current at two load buses and assumes a known admittance between the two. To overcome the problem of having nonlinear and dynamic loads being lumped with the system equivalent, reference [11] proposes a network decoupling transform which models the effect of all the other loads as constant virtual impedance. The result is a nearly constant Thévenin impedance which does not change in the absence of PV-PQ transitions in the system generators. Reference [12] provides an exhaustive list of different technique used for voltage instability detection. As an outgrowth of the results presented in [13], this work is aimed at studying the changes in the distribution of the VIP locations across a given network in terms of their accuracy of margins. The system conditions are synthetically varied by increased system loading and/or by randomly changing the status of Over Excitation Limiters 
(OELs) thus inducing PV-PQ transitions at different voltage levels and in different locations of the network. In the absence of analytical proof of the concept, such experiments greatly increase trust in the validity of the proposed methodology.

\section{Stability Margin Estimation}

VIP models the external system seen from the point of measurement as a Thévenin equivalent. The two equivalent parameters are the Thévenin emf, $E_{T h}$ and Thévenin impedance, $Z_{T h}$. Once the external system parameters are identified, metrics for distance from the operating point to the point of collapse can be determined. In particular, the loading margin can be derived from the two system equivalent parameters. Although VIP suffers from some identification problems, particularly in the estimation of the model parameters, such problems are overcome by sampling discrete time sequence of voltage and current phasors. Between two sufficiently close sampling events, system parameters show nearly constant characteristics in the absence of topology-changing events such as equipment outages of generator PV-PQ transitions. The assumption of constancy of the Thévenin model allows parameter identification and facilitates determination of the system equivalent impedance and voltage. The length of the data window chosen for sampling the time sequence measurements of voltage and current phasors is critical for optimal estimation of model parameters. The measurements are sampled at time instants or loading instants, which allow a change in the operating point while at the same time maintaining the quasi-constant nature of the system equivalent. Achieving this tradeoff between sufficiently closely spaced sampling instants and measurably different system conditions is critical in obtaining meaningful information from the measurements.

Margin quantitatively defines the relationship between the distance to a possible voltage collapse scenario and the current system loading. The VIP method, introduced in [6], offers a possibility to quantify the distance to collapse in terms of system loading as a percentage of base load. As the system is progressively stressed through the use of a load multiplier, $\lambda$, the apparent load impedance moves closer to the Thevenin impedance becoming equal to Thevenin Impedance in magnitude at the point of collapse. Quantitatively at the point of collapse for a constant power load the following relationship holds true

$$
\left|Z_{L}\right|=\left|Z_{T h}\right|
$$

where $\left|Z_{L}\right|$ represents the magnitude of the load impedance and $\left|Z_{T h}\right|$ is the magnitude of the Thevenin impedance. As a result of this, the difference between two impedances can serve as an indicator of the available system margin in the direction of the assumed system loading. Equation (1) can be used to derive the expression for maximum power that can be delivered to a load bus. Since margin at any arbitrary system loading, $\lambda$, is defined as

$$
\Delta S=S_{\max }-S_{\lambda}
$$

Where $S_{\max }$ is the maximum deliverable power at a bus and $S_{\lambda}$ is the complex power injection to the load bus at a system loading of $\lambda$, a measure of system margin in terms of apparent power is possible. The margin thus obtained has to be mapped in terms of system loading for a system wide comparison with other VIP locations. If $M_{\lambda, j}$ represents the system margin in terms of system loading at bus ' $j$ ' at a loading factor $\lambda$, and $\Delta S_{j}$ represents the margin in terms of apparent power at bus ' $j^{\prime}$, where $j \in\left\{P Q_{\text {buses }}\right\}$, then

$$
M_{\lambda, j}=\frac{\Delta S_{j}}{S_{j, 0}}
$$

Where $S_{j, 0}$ represents the base load of bus $j$.

Figure 1 shows a power system being represented by a two bus Thévenin equivalent connected to the load bus.

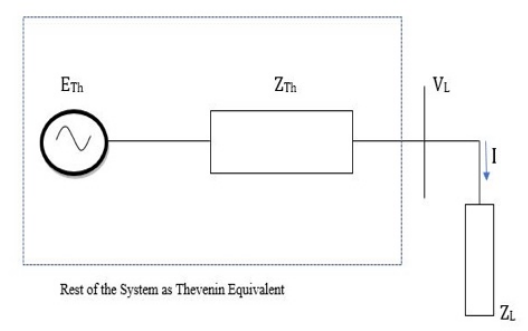

Figure 1. Local bus and Rest of the System treated as Thevenin Equivalent

The current $\bar{I}$ in Figure 1 is given by

$$
\bar{I}=\frac{\bar{E}_{T h}}{\left(R_{T h}+R_{L}\right)+j\left(X_{T h}+R_{L} \tan \phi\right)}
$$

Where $R_{T h}$ and $X_{T h}$ are the real and imaginary parts of the Thevenin Impedance, $R_{L}$ is the real part of the load impedance and $\phi$ is the load power factor angle. At the point of voltage collapse the extremum condition can be stated as

$$
\frac{\partial P}{\partial R_{L}}=0
$$

This leads to the equation for the maximum power in terms of the Thevenin parameters of the system as defined by (6). 


$$
S_{j, \text { max }}=\frac{E_{T h, j}{ }^{2}}{2} \frac{1}{\left[Z_{T h, j}+\left(R_{T h, j} \cos \phi_{j}+X_{T h, j} \sin \phi_{j}\right)\right]}
$$

Where $S_{j, \max }$ is the maximum power that could be delivered at bus $j \in\{P Q\}, E_{T h, j}$ is the magnitude of the Thevenin model emf at bus $j, Z_{T h, j}$ is the magnitude of the Thevenin Impedance at bus $j, R_{T h, j}$ and $X_{T h, j}$ are the real and imaginary parts of the Thevenin Impedance at bus $j$ and $\phi_{j}$ is the load power factor angle at bus $j$. The maximum deliverable power given by equation (6) is a function of the system loading parameter, $\lambda$. An alternate expression in terms of active power can be written as

$$
P_{j, \text { max }}=\frac{\left|E_{T h, j}\right|^{2} \operatorname{Re}\left(\frac{Z_{L, j}}{\left|Z_{L, j}\right|}\left(\left|Z_{T h, j}\right|\right)\right)}{\left|Z_{T h, j}+\frac{Z_{L, j}}{\left|Z_{L, j}\right|}\right| Z_{T h, j}||^{2}}
$$

Where $P_{j, \max }$ is the maximum real power transferable to Bus $j$, while $Z_{L, j}$ and $Z_{T h, j}$ represent the load impedance and the corresponding Thévenin impedance at bus $j$. Again, active power margin can be trivially derived from equation (7) in a fashion similar to equation (2), as

$$
\Delta P_{j}=P_{j, \max }-P_{\lambda}
$$

Where $\Delta P_{j}$ is the available active power margin at Bus $j$, and $P_{\lambda}$ is the active power injected at Bus $j$ at system loading $\lambda$. It is important to note that power margins given by Equations (2) and (8) are implicit functions of system loading parameter, $\lambda$. In fact, margin is a monotonically linearly decreasing function of system loading factor marked by sharp discontinuities at the instants which correspond to generators reaching their respective reactive power limits. When a generator reaches the reactive limit, it loses control of its terminal voltage and switches from a voltage controlled (PV) bus to a voltage variable (PQ) bus. Such generator switching instants are observed through discontinuous changes in the system Thévenin equivalent parameters. Since system margin is derived by extrapolating the impedance trajectories of system Thévenin impedance and load impedance, such events of PV-PQ transitions significantly, impact the VIP-derived margin. In a theoretical sense, margins obtained at all the load buses should agree with each other. However, the estimated margins obtained from VIP are not equal, largely due the heuristic nature of the VIP algorithm. Therefore, if an aggregation of wide area margin estimates from local VIPs is possible, useful information to improve the accuracy of margin estimation could be extracted from the redundant margin estimates throughout the network.
The impedance matching condition proposed by the VIP in equation (1) at the point of collapse is a direct consequence of

$$
\frac{d P}{d V_{j}}=0
$$

Where, $V_{j}$ is the magnitude of the voltage at bus $j$, at the point of collapse. If the load power factor at bus $j$ is $\cos \phi_{j}$, active power can be written as

$$
P_{j}=V_{j} I_{J} \cos \phi_{j}
$$

The load current, $I_{J}$ is a function of bus voltage, $I_{J}=I_{J}\left(V_{j}\right)$, hence the derivative of $P_{j}$ with respect to the bus voltage yields

$$
\frac{d P}{d V_{j}}=\left(I_{j}+V_{j} \frac{d I_{j}}{d V_{j}}\right) \cos \phi_{j}
$$

At the point of voltage collapse, which corresponds to the nose of the PV curve for a constant power load, equation (10), further yields

$$
\frac{d I_{j}}{d V_{j}}=-\frac{I_{j}}{V_{j}}
$$

Equation (11), reiterates the statement of equation (1), which essentially derives from the fact that, at the point of collapse, and for a constant power type of load, $\frac{d P}{d V_{j}}=0$. Since margin is a linearly decreasing function of the system loading, the maximum power transferable to a bus as given by equations (6) and (7), should turn out to be constant. However, due to nonlinearity inherent in the Thévenin parameter identification, the maximum transferable power varies with the system loading. As the estimated Thévenin model parameters approach their true value, the maximum power calculated using equations (6) and (7) also approaches its true value. This explains the convergence of the VIP margins as the system evolves towards an impending instability. In this paper, a proportional increase in the system loading is assumed, (active as well as reactive load), preserving constant load power factors. The sampling instants are chosen in simulations such that the system load is increased in steps of $0.05 \%$, which allows for a change in the system operating point while also maintaining the quasiconstant characteristics of the Thévenin model parameters. The active and reactive loads at bus $j$, at any arbitrary system loading level, $\lambda$, can be written as

$$
\begin{aligned}
P_{D, j}(\lambda) & =\lambda P_{D, j}^{\prime} \\
Q_{D, j}(\lambda) & =\lambda Q_{D, j}^{\prime}
\end{aligned}
$$

Where $P_{D, j}^{\prime}$ and $Q_{D, j}^{\prime}$ are the active and reactive loads on bus $j$, at base load i.e corresponding to $\lambda=1$. The standard IEEE 9 bus system, as shown in Figure 2, illustrates the spread of the estimated VIP margins (at 
different locations) and the evolution of the margins under the condition of increased system loading. The arrows on the buses mark the VIP locations. Figure 3 plots the VIP derived margins of load buses. To inject some degree of redundancy, additional load is placed at Bus 8 which leads to four VIP locations in this network.

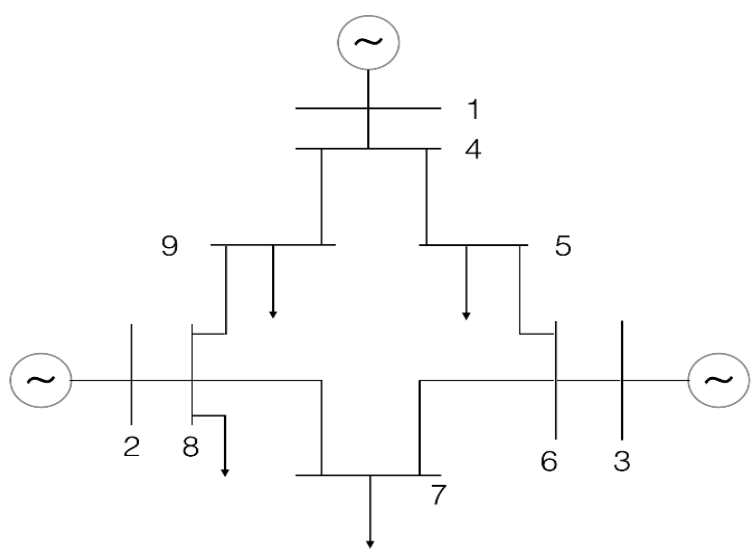

Figure 2. Example 9 Bus System.

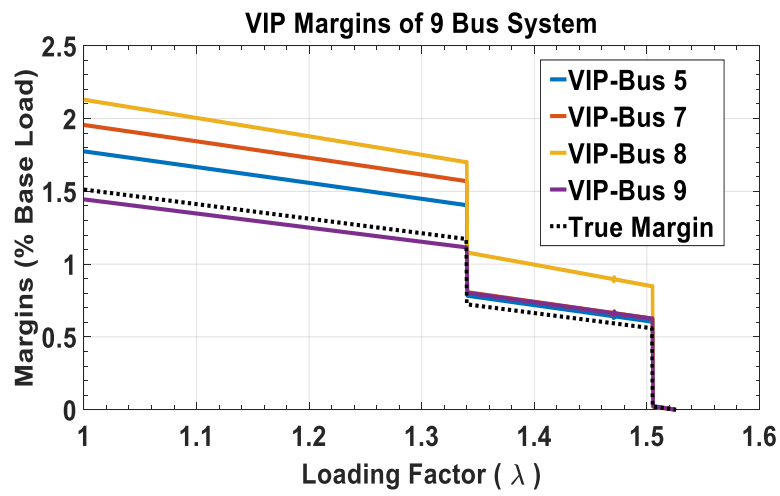

Figure 3. VIP Margin Distribution of 9 Bus System.

\section{VIP Margin Accuracy}

The inaccuracies inherent in the Thévenin parameter identification, due to the nonlinear characteristic of the system, are reflected in the VIP margins in the form of a distribution around the true system margin. In equations (6) and (7), the values of the Thévenin model parameters correspond to their values at critical point. However, the assumption of constancy of the Thévenin equivalent makes it possible to use the present estimated values of the Thévenin equivalent in order to yield a measure of the power injected at a bus at the critical loading factor. The assumption of constancy works well within a sampling window of sufficiently short length. When spread over the entire loading space, which may include an arbitrary number of PV-PQ transitions, system parameters tend to gradually drift from their initial values. This small but a gradual drift in the system parameters offsets the estimation of the true maximum power. Depending on the drift experienced by the VIP locations, the accuracy of VIP margins changes. In Figure 3, the VIP location bus 9 is consistently more accurate than the rest of the VIP locations with VIP on Bus 8 being the least accurate. Figure 4 plots the relative drift in the Thévenin Impedance experienced by VIP locations from system base load which corresponds to, $\lambda=1$ to the critical loading factor of the system corresponding to, $\lambda \cong 1.5261$.

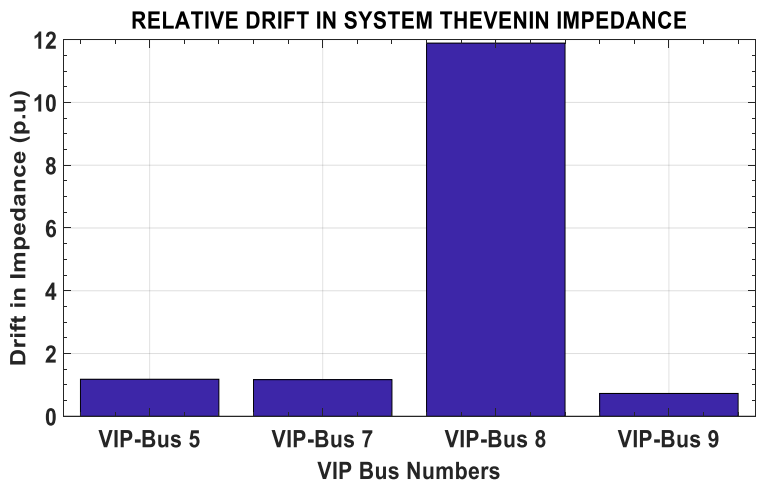

\section{Figure 4. Changes in the System Thévenin Impedance on different VIP Locations.}

A wide variety of changes in the external system conditions can lead to large changes in the parameters of the Thévenin model. Apart from system loading, which is normally the dominant factor in determining the VIP margins, factors such as the status of OELs of generators also greatly impacts the values and changes of VIP margins. In addition, contingencies like line outages also lead to a change in the estimated margins at loads and thus creating a different distribution of VIP margins. In view of these observations, it becomes very difficult to predict a particular VIP location in order to reveal the information about available system margin. Whenever any generator reaches the reactive limit, it represents a changed system condition, making it necessary to reevaluate the accuracy ranking of the VIPs. Similarly, line outages cause a change in system topology, which negatively impacts the available system margin and changes the order of the accurate VIP locations. In this work, VIP margin accuracy is assessed by manually altering the reactive limits of the generators, thus creating a distinct pattern of PV-PQ transitions.

\subsection{Effect of Generator Reactive Limits}

The standard IEEE 9 bus system as shown in Figure 2 is studied under a different a set of generator reactive limits and the related impact on the VIP derived margins. The standard 9 bus system is equipped with three generators, including the slack bus, and three 
loads to which one more load is added for the purposes of having redundant measurements. Three different cases are created, which correspond to any one of the generators reaching its limit or both generators reaching their respective limits. The load on Bus 8 is varied proportionally in the three cases along with the generator reactive limits, to create a distinct loading profile for each case, which allows the possibility of different PV-PQ transitions. It is to be expected that when two generators, with the exception of the swing bus, are allowed to reach their limits, the impact on the system margin and the accuracy spectrum of VIPs will be significant as opposed to only one generator reaching the limit. For the sake of simplifying the analysis, different loading factor regions are created. Since the system behavior remains almost unchanging between two successive PV-PQ transitions, it is important to evaluate the margins in such regions, separated by consecutive PV-PQ transitions. A generator switching from PV mode to PQ mode signifies a precipitous decline of the reactive reserves of the system, while also presenting an extra burden to the system. In the absence of any emergency controls or reactive compensation at buses, PV-PQ transitions can effectively drag the system into collapse. However, it has also been observed that generators can switch from PQ to PV mode, which signifies an increase in the reactive reserves of the system. Such switches have been observed to have an effect of increased margin available in the network. Under any such transitions, the accuracy of a VIP margin depends on the accuracy of estimation of $S_{j, \max }$ or $P_{j, \max }$. Load buses, which are able to estimate them as close as possible to their true values, are naturally associated with better accuracy in their margin estimation.

In Figure 2, bus 1 is the slack bus while buses 2 and 3 are voltage-controlled buses. The first case is created by manipulating the reactive limits of the generators in such a way that allows only generator-2 to reach its limit, thus changing from a PV (voltage-controlled) bus to a PQ bus. Table 1 lists the data for this case and the associated accuracy ranking of VIP locations.

Table 1. Only Gen-2 reaches reactive limit.

\begin{tabular}{|c|c|c|c|}
\hline \multirow{2}{*}{ Gens } & $\begin{array}{c}\text { Q Limit } \\
\text { (MVAR) }\end{array}$ & \multicolumn{2}{|c|}{$\begin{array}{c}\text { Accuracy Ranking of VIP } \\
\text { Locations }\end{array}$} \\
\hline $\begin{array}{c}\text { Gen-1 } \\
\text { (Slack) }\end{array}$ & 400 & $\begin{array}{c}\text { Region-I } \\
\lambda=1 \sim 1.68\end{array}$ & $\begin{array}{c}\text { Region-II } \\
\lambda=1.68 \sim 1.869\end{array}$ \\
\hline \multirow{2}{*}{ Gen-2 } & 100 & Bus-9 & Bus-8 \\
\cline { 3 - 4 } Gen-3 & 300 & Bus-5 & Bus-9 \\
\cline { 3 - 4 } & & Bus-8 & Bus-7 \\
\hline & & \multicolumn{2}{c}{ Bus-5 } \\
\hline
\end{tabular}

Figure 5 shows the estimates of the margins for the case when only generator- 2 reaches the reactive limit. A significant change in the accuracy spectrum results when generator at bus 2 reaches the limit. Before the PV-PQ transition, the VIP margin of Bus 8 is characterized by highest inaccuracy.

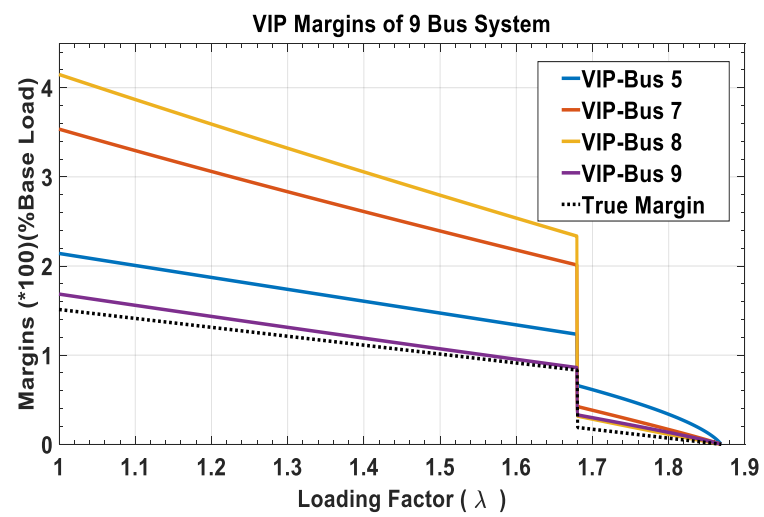

Figure 5. VIP Margin Distribution of 9 Bus System when Gen-2 reaches the reactive limit.

The switching of generator 2 also has a huge impact on the system margin. The available margin then undergoes a drastic change and drops by more than $50 \%$. The critical loading factor of the system is $\lambda_{c} \cong$ 1.869. In the second case, the generator at bus 3 is allowed to reach its reactive limit and switch from PV mode to PQ mode, while the generator at bus 2 stay in PV mode. Table 2 lists the data for this case and the associated VIP margin accuracy ranking.

Table 2. Only Gen-3 reaches reactive limit.

\begin{tabular}{|c|c|c|c|}
\hline Gens & $\begin{array}{c}\text { Q Limit } \\
\text { (MVAR) }\end{array}$ & \multicolumn{2}{|c|}{$\begin{array}{c}\text { Accuracy Ranking of VIP } \\
\text { Locations }\end{array}$} \\
\hline $\begin{array}{c}\text { Gen-1 } \\
\text { (Slack) }\end{array}$ & 500 & $\begin{array}{c}\text { Region I } \\
\lambda=1 \sim 1.65\end{array}$ & $\begin{array}{c}\text { Region II } \\
\lambda=1.65 \sim 2.161\end{array}$ \\
\hline Gen-2 & 355 & Bus-7 & Bus-9 \\
\hline Gen-3 & 50 & Bus-5 & Bus -7 \\
\hline & & Bus-9 & Bus-5 \\
\hline & & Bus-8 & Bus-8 \\
\hline
\end{tabular}

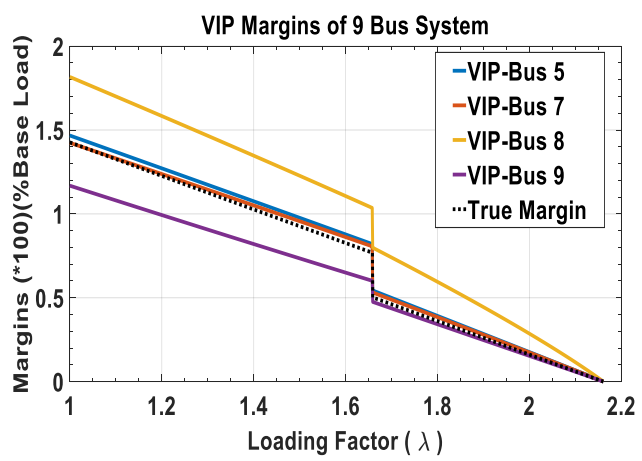

Figure 6. VIP Margin Distribution of 9 Bus System when Gen-3 reaches the reactive limit. 
Figure 6 plots the VIP margins when only generator3 is allowed to reach the reactive limit. Figures 5 and 6 can be compared and the differences in the VIP margins are apparent.

The VIP margins of Figure 6 show a markedly different distribution around the true margin with different accuracy ranking as is shown in Table 2 . The critical loading factor in this case turns out to be $\lambda_{c} \cong 2.161$. The apparent increase in the system critical loading factor can be attributed to the increase in the reactive reserves of the system. By allowing a different generator to reach the reactive limit, a different spread of VIP margins was obtained. In reality, generators have fixed reactive limits, which are independent of system loading, however this experiment reveals the margin tracking capability of the VIP algorithm, as well as its locational dependence of accuracy of margin estimates. In the third and final case, both generators are allowed to reach their respective reactive limits and a different distribution of the VIP derived margins is obtained. Table 3 lists the data for this case and Table 4 lists the associated VIP margin accuracy ranking.

Table 3. Both Gens reach reactive limit.

\begin{tabular}{|c|c|}
\hline Generator Bus & $\begin{array}{c}\text { Reactive Limit } \\
\text { (MVAR) }\end{array}$ \\
\hline Generator-1 (Slack Bus) & 400 \\
\hline Generator-2 (PV Bus) & 75 \\
\hline Generator-3 (PV Bus) & 20 \\
\hline
\end{tabular}

Table 4. VIP Margins Accuracy Ranking when both generators reach reactive limits.

Accuracy Ranking of VIP Locations

\begin{tabular}{|c|c|c|}
\hline $\begin{array}{c}\text { Region I } \\
\lambda=1 \sim 1.14\end{array}$ & $\begin{array}{c}\text { Region II } \\
\lambda=1.14 \sim 1.15\end{array}$ & $\begin{array}{c}\text { Region III } \\
\lambda=1.15 \sim 1.26\end{array}$ \\
\hline Bus 7 & Bus 8 & Bus 7 \\
\hline Bus 5 & Bus 9 & Bus 8 \\
\hline Bus 9 & Bus 7 & Bus 9 \\
\hline Bus 8 & Bus 5 & Bus 5 \\
\hline
\end{tabular}

As both generators are allowed to reach their respective limits in this example, the system approaches instability faster, and voltage collapse occurs at a system loading factor of $\lambda_{c} \cong 1.26$. This also changes the pattern of margin spread around the true margin and the new accuracy rankings are listed in Table 4. Since the impact on margins is significant, it becomes necessary to study some of the easily measurable electrical properties of the buses, which change in accordance with the change in the VIP margins as system operating point moves through different conditions. Any such property should have the feature of being easily measurable at every system loading factor and should be able to reflect the changes in the VIP margins with a significant amount of fidelity. It could then provide an easy access in to the changes that happen to the bus margins, under events of contingencies or any future PV-PQ transitions or even under heavily stressed conditions. By monitoring such a property, the accuracy of the VIPs could be easily established with a higher degree of certainty. Figure 7 shows the VIP margins when both generators are allowed to reach their limits. Note the difference in the spread of VIPs around the true margin.

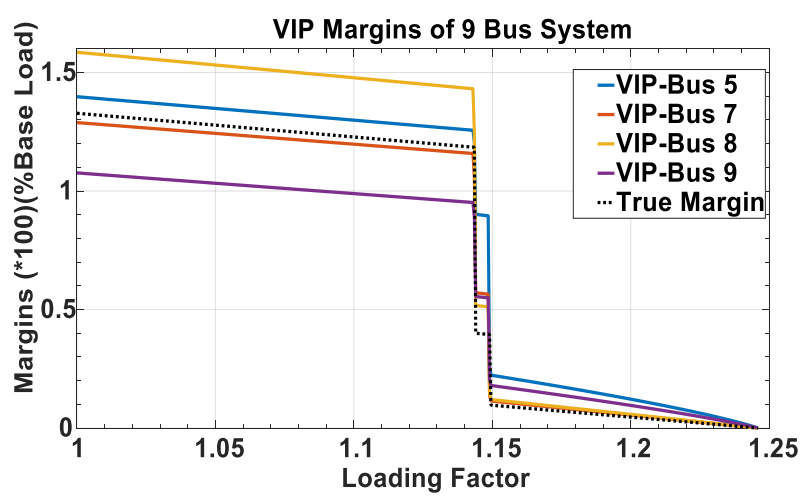

Figure 7. VIP Margin Distribution of 9 Bus System when Gens 2 and 3 (both) reach the reactive limit.

\section{Characterization of VIP Margins}

The dependence of the calculated VIP margins w.r.t. changing system conditions necessitates some form of locational characterization. We are seeking electrical properties which correlate well with margin accuracy to be able to rank margin estimates. However, due to the nonlinearity of the system and the difficulties associated with extrapolating the Thévenin parameters near the critical point, a direct correspondence between the VIP margin accuracy and any such electrical property may be hard to find. The limitations imposed by the nonlinearity of the system can be overcome by exploiting the redundancy of data that is available in a VIP network. In this sense the multiplicity of data becomes an asset which if manipulated in a meaningful manner, can be used to offset the liability; which is the in deterministic nature of the VIP algorithm. To allow for locational characterization of the VIP margins, a sensitivity metric is proposed for monitoring the local sensitivity of VIP locations with respect to the change in local load. The sensitivity metric is defined as 


$$
K_{j}^{\lambda}=\frac{\delta V_{j}^{\lambda}}{\delta S_{j}^{\lambda}}=\frac{\frac{V_{j}^{\lambda}-V_{j}^{\lambda-1}}{V_{j}^{\lambda}}}{\frac{S_{j}^{\lambda}-S_{j}^{\lambda-1}}{S_{j}^{\lambda}}}
$$

Where $K_{j}^{\lambda}$ is the measure of the sensitivity metric at bus $j$ at system loading $\lambda$ while $\frac{\delta V_{j}^{\lambda}}{\delta s_{j}^{\lambda}}$ refers to the ratio of percentage change in the voltage at bus $j$ with respect to the percentage change in the complex load at bus $j$, between two consecutive instants of system loading. The percentage change in the voltage and in the local load is used for normalization, because different VIP locations have different amounts of loads present on them. Local sensitivity of VIP locations can be easily determined, and the computation can be performed for every loading factor. This has the benefits of being able to assign confidence degrees to the individual VIP margins as the system is proportionally loaded from base load until collapse point. The use of the confidence degrees can be further exploited to yield a meaningful data fusion of the VIP margins. Since each VIP margin carries some degree of uncertainty, a fusion of a set of margins can attempt to reduce the entropy of the individual margins and can possibly yield a better estimate of the system margin. Of course, in order for data fusion to produce meaningful results, more accurate VIP locations need to identified and isolated from the less accurate VIP locations. This process of elimination of the less accurate margins needs to be performed at every measurement point. The robustness of the sensitivity metric to filter out the less accurate margin estimates is contingent on accuracy with which the Thevenin parameters are estimated. In a number of experiments that have been performed, the local sensitivity of the VIP margins at different locations, as given by equation (14), has shown a correlation with the accuracy of VIP margins. Often assuming the shape of a decreasing exponential function, the VIP locations that are characterized by higher values of the sensitivity metric turn out to be more accurate than VIP locations with low values of the sensitivity metric. Furthermore, as we move towards the higher end of the sensitivity spectrum, the number of such locations decreases, indicating that a significant number of the locations are characterized by low sensitivity measures which agrees with the behavior of accuracy spectrum of the VIP margins.

IEEE-118 bus system is used to demonstrate the correlation between the local sensitivity of the VIP margins and the corresponding accuracy. Figure 8 draws the correlation between the two quantities of sensitivity and accuracy at base load, $\lambda=1$ and near critical load, $\lambda=2.109$. From Figure 8 it can be concluded that as the system is progressively stressed, the correlation between the VIP accuracy and the VIP sensitivity grows stronger. At the base load the spread is over a wider region but is distributed over a narrow region near the critical load. This suggests that as the system moves closer to the collapse, the accurate VIP locations tend to get more sensitive. This observation can be exploited at every instant of system loading and the accurate VIP locations can be identified throughout the network.
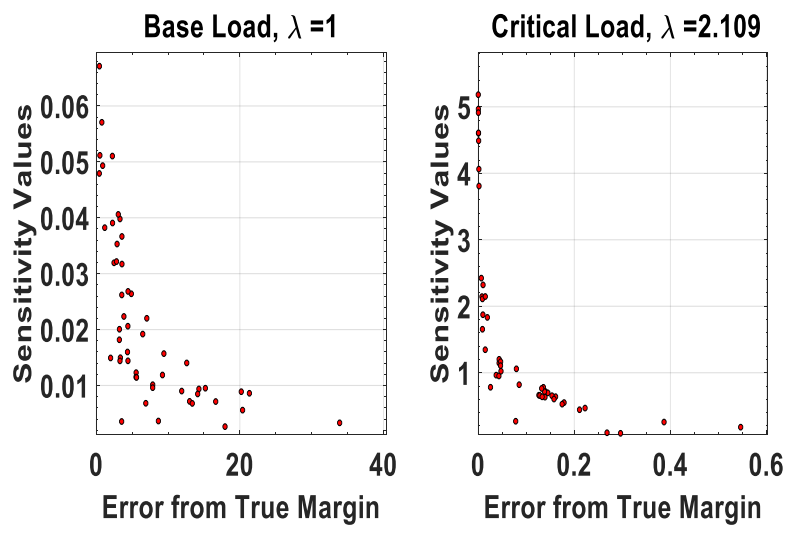

Figure 8. Correlation between VIP accuracy and VIP sensitivity at $\lambda=1$ and $\lambda=2.109$

The buses with the largest sensitivities are referred to as critical buses as a small increment in the load power demand can cause the system state to approach instability, in the absence of some control action. In [15], the identification of critical locations for reactive power support are based on the sensitivities of the total generated reactive power to load powers. It is shown that by monitoring the most sensitive locations, voltage stability assessment can be performed with excellent approximation. The proximity indicator (margin function) converges to zero at the point of saddle-node bifurcation. In case of a proportional load increase, the system state $(\theta, V)$ undergoes a gradual change under the influence of system loading parameter $(\lambda)$. Near the point of instability

$$
\lim _{\lambda \rightarrow \lambda_{c}} M(\theta, V, \lambda)=0
$$

The critical nodes in a power system as defined by their sensitivities affect the onset of voltage collapse dramatically. Since the instability of the operating point is caused, among other reasons, by the deficiency of reactive power, incremental increase in the load demand at such locations close to critical point requires a steep elevation in the reactive power generation. The critical loads in a power network can steer the system out of a near-unstable situation much more effectively, when reactive compensation is used at such points. For critical loads, 


$$
\left\|P_{j} \frac{\partial M}{\partial P_{j}}+Q_{j} \frac{\partial M}{\partial Q_{j}}\right\| \geq \epsilon
$$

the condition (16) is met which isolates the load points with largest sensitivities. In case of large power systems, the authors in [16] propose a coherency criteria and calculate the sensitivities of the total generated reactive power with respect to cluster loads and inter-cluster power transfers. By employing the clustering technique a set of coherent buses is obtained, where the voltage phasors obey some pre-defined coherency criteria. The results of the paper indicate excellent approximation to the assessment of voltage stability by monitoring the minimum singular value of the approximate Jacobian. The approximate Jacobian is calculated from the reduced vector where voltage phasors belonging to a particular cluster are assumed to be equal.

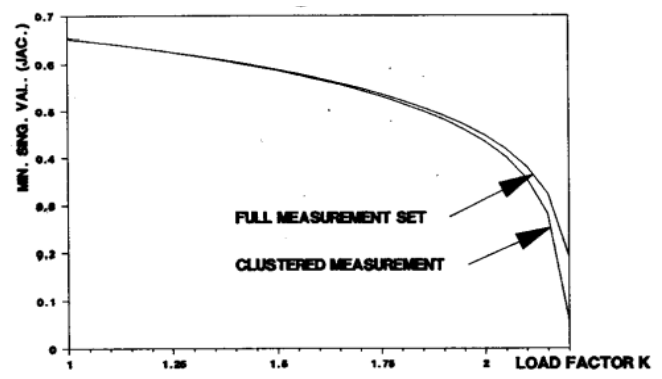

Figure 9. Minimum Singular Value of accurate and approximate Jacobians by monitoring sensitivities of clustered loads [16].

Since the method of VIP is based on a somewhat inaccurate modelling of a non-linear network, it is very difficult to obtain the true Thevenin parameters near unstable situations at a relatively early period through extrapolation. The heuristic nature of the algorithm produces a diverse distribution of the stability margins. The inaccuracies encountered in the identification of Thevenin equivalent are further compounded in the derivation of stability margins by the assumption of constancy of Thevenin equivalent.. In such a situation an analytic solution to the problem is extremely difficult, if not impossible. However in the future, a closed form solution of a small power network with few VIP points with less non-linearity will be attempted.

\section{Simulations}

The IEEE-118 Bus System is subjected in this simulation to a series of different tests for studying the spectrum of VIP margins, the changes caused by different system conditions and for validating the effectiveness of the sensitivity metric in its ability to identify the most accurate VIP locations. A total of 100 test cases have been created, each with a unique combination of the generators that reach the respective reactive limits. By allowing a different combination of generators to reach the reactive limit enough diversity is created in the system in order to empirically test the validity of the sensitivity metric. Each case is characterized by a unique PV curve with distinct instants of PV-PQ transitions. A combination of five such generators is taken in each case and their reactive limits are altered to ensure that they reach their limits. Since the IEEE-118 Bus System has 54 thermal units, a huge number of combinations is possible depending on the sample size taken. To limit the number of combinations and make it more manageable, a sample of five generators is taken. It has been observed that a sample of five generators generates sufficient diversity in the spectrum of PV-PQ transitions. In a similar fashion different sets of PV-PQ transitions are simulated and the impact on the VIP margins is analyzed. The process of drawing combination is done in the following manner:

- The standard IEEE-118 Bus case is simulated, without any modifications, under a proportional load increase.

- The generator that do not reach their reactive limits at the critical load are identified. Such generators are $24,25,26,27,31,40,42,61$, $66,69,72,73,87,89,90,91,107,111,112$, $113,116$.

- Combinations of five generators are drawn out of the set of generators that do not reach their reactive limits by making use of $\frac{n !}{(n-k) ! k !}$, where $n$ is the number of generators in PV mode at the critical load and $k$ is the sample size, which in this case is 5 .

- The sample size could be adjusted, increased or decreased, depending on the number of combinations sought. However, a large sample size may result in numerical problems in the power flow solutions, because of forcing a large number of generators to switch at once, and a sample size of less than five may not generate sufficiently diverse combinations.

- A sample size of five results in 20349 combinations, out of which 100 very diverse combinations are selected. The 100 combinations that are selected are the combinations of generators which lie in the vicinity of heavy loads (Buses 66-118).

By simulating a particular combination of generators to reach the reactive limits, a unique set of PV-PQ transitions is obtained. It is important to note that in each case, in addition to the generators which are 
forced to switch, other generators will also reach their limits as system loading is varied. Every new combination presents a changed system condition with a distinct PV curve. The most accurate VIP location is tracked in each case. As different generators switch from PV mode to PQ mode, the critical VIP locations travel across the system and the accuracy spectrum changes with every new PV-PQ transition. Figure 10 shows some of the common VIP locations that are most accurate across 100 test cases. It is clear from Figure 10 that there is no single location that is the most accurate across all the test cases. In fact, the most accurate location keeps on changing and moves across the entire system under a changing set of PV-PQ transitions. IEEE-118 bus system has a total of 53 possible VIP locations.

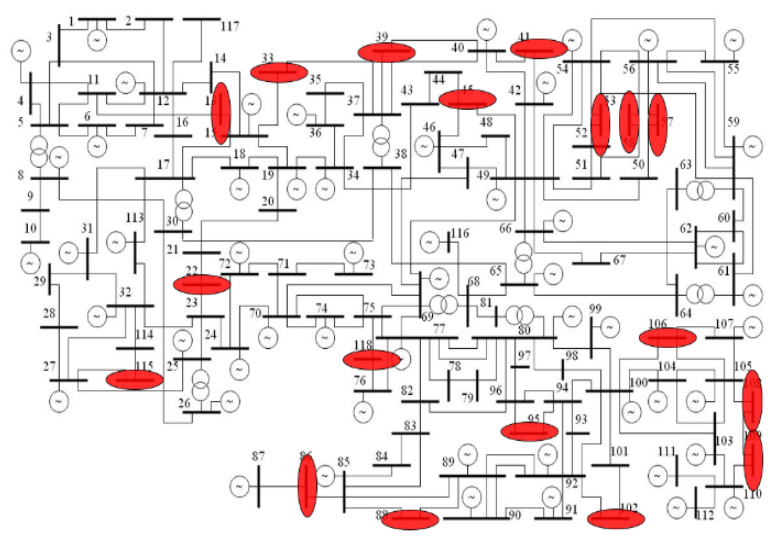

Figure 10. VIP locations that are commonly most accurate across 100 test cases.

The sensitivity metric is employed for measuring the local sensitivities of the VIP locations and the sensitivity bounds of top $5 \%$, top $10 \%$, top $20 \%$, top $30 \%$, top $40 \%$, top $50 \%$ and top $60 \%$ are created and the correlation with similar bounds of accuracy is studied. The 5\% sensitivity bound includes the top 5\% most sensitive VIP locations and so on. As the sensitivity thresholds are increased, thus encapsulating more buses, the corresponding correlation with a similarly increased accuracy threshold is verified. This is important for two reasons. First, increasing the bounds and studying the correlation for each bound would shed light on the optimum size of the sensitivity threshold and second, such an analysis could be helpful in revealing the number of outliers that appear in each threshold. In [14], the idea of fusion of margins based on Dempster-Shafer evidential reasoning is explored. In this work, fusion of margins is attempted and the percentage error between the true system margin and the fused margin is observed for different bounds of sensitivities.

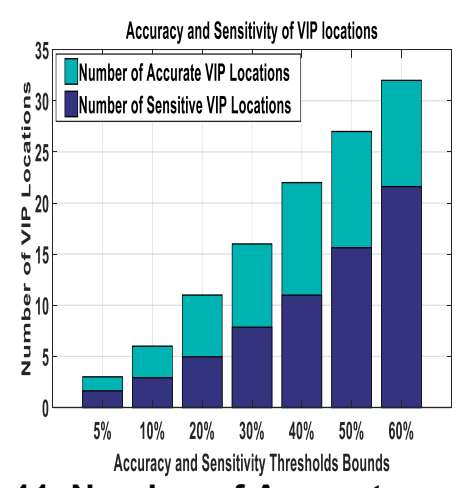

Figure 11. Number of Accurate and Sensitive VIP Locations (Average for 100 cases)

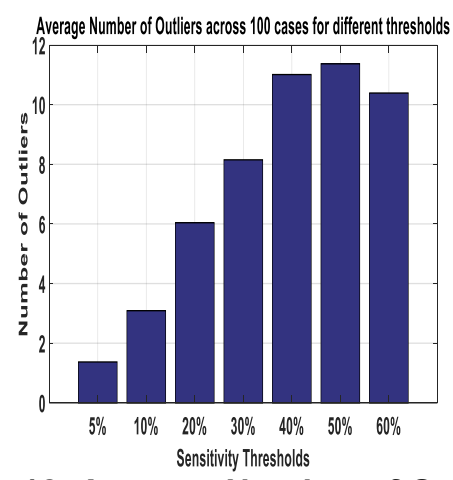

Figure 12. Average Number of Outliers for each threshold.

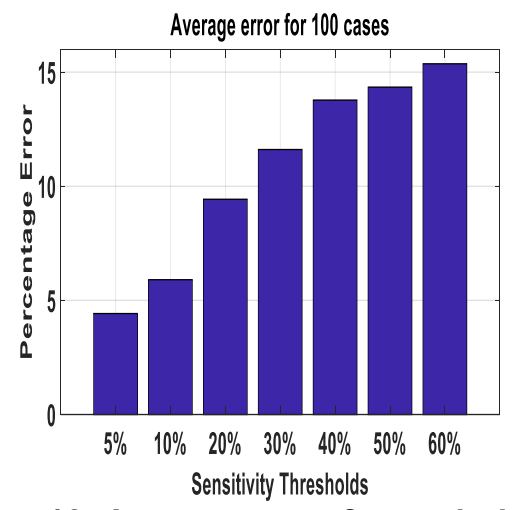

Figure 13. Average error for each threshold across 100 cases.

The bound which results in strongest correlation and least error represents the number of sensitive VIP locations that should be retained for fusion. It is important to note the results in this section are representative of the state of the system when the available margin is $30 \%$. Figure 11 plots the correlation or similarity between different thresholds of accuracy and sensitivity of VIP locations. It is clear that as the thresholds are relaxed, the number of outliers increases as is shown in Figure 12. Since the implementation of DS fusion in this work is not 
resilient to the presence of outliers in the collected data, the error between the fused margin and the true margin invariably increases as the thresholds limits are increased. The results presented in Figures 11, 12 and 13 are average results for 100 cases. A sensitivity threshold of 5\% seems to produce least amount of error across 100 cases, only slightly more than $10 \%$ sensitivity limit. This results are encouraging in the sense that it mandates the monitoring of only a small number of VIP locations in a given network. Figure 13 shows that by retaining the most $5 \%$ sensitive buses, the average error between the fused margin and the true margin is less than $5 \%$ from the loading instant when the available system margin is $30 \%$ of the base case loading.

\section{Conclusions}

The method to calculate the VIP-based estimated margins is presented. It is shown how the locational accuracy and evolution of the margins is affected by the changing system conditions, especially voltage sensitivities at measurement locations. The synthetic simulated PV-PQ transitions radically alter the system state, thus causing a shift in the margin accuracy spectrum. A sensitivity metric is proposed to allow for locational characterization of the VIPs. The robustness of the metric is tested by creating a large number of different test cases of the IEEE-118 bus system, each with a unique combination of generators reaching the reactive limits.

The method of estimating the VIP margins is being enhanced by identifying a subset of measurements, consisting of the most accurate VIP locations, by using their voltage sensitivities w.r.t. load changes (easy to calculate local measurements, just like those used in determining the VIPs). More diversity in the experiments increases the confidence level of the proposed VIP margin calculations. The opportunities for an effective data fusion of the VIP margins are currently being explored and the issues of scaling up to applications in large systems are being explored. The modification of VIP margin calculations presented in this paper allows to identify a subset of the most accurate margin estimates and use them for subsequent estimate of the true margin by using the discrete distribution thus obtained, potentially greatly improving both speed and accuracy of the margin estimates while maintaining the simplicity of using local measurements and limited communications for all but the final fusion of margin estimates.

\section{References}

[1] I. Dobson and H. D. Chiang, "Towards a Theory of Voltage Collapse in Electric Power Systems," Systems \& Control Letters 13, 1989, pp. 253-262.

[2] E. H. Abed, et al., "On bifurcations in power system models and voltage collapse," in Proc. 29th Conf. Decision and Control, pp. 3014-3015,1990.

[3] E H Abed, J C Alexander, H Wang, A M A Hamdan, and $\mathrm{H}-\mathrm{C}$ Lee, "Dynamic bifurcations in a power system model exhibiting voltage collapse," Int. J. Bifurcations Chaos, vol. 3, pp 1169-1176, 1993.

[4] H. D. Chiang, C W Liu, P. P. Varaiya, F. F. Wu, and M G Lauby,"Chaos in a simple power system," IEEE Trans Power Systems, vol. 8, no. 4, pp. 1407-1417, Nov, 1993.

[5] C. W. Taylor, Power System Voltage Stability, EPRI Power System Engineering Series, McGraw Hill, 1994.

[6] K. Vu, M. M. Begovic, D. Novosel, and M. M. Saha, "Use of local measurements to estimate voltage-stability margin," Power Systems, IEEE Transactions on, vol. 14, no. 3, pp. 1029-1035, 1999.

[7] B.Milosevic and M. Begovic, "Voltage-stability protection and control using a wide area network of phasor measurements," Power Systems, IEEE Transactions on, vol. 18, no. 1, pp. 121-127, 2003.

[8] S. Corsi and G. N. Taranto, "A real-time voltage instability identification algorithm based on local phasor measurements," IEEE transactions on power systems, vol. 23, no. 3, pp. 1271-1279, 2008.

[9] C. D. Vournas, T. van Cutsem, "Local Identification of Voltage Emergency Situations," IEEE Trans. Power Syst., Vol. 23, No. 3, pp. 1239- 1248, Aug. 2008.

[10] L. Warland and A. T. Holen, "A voltage instability predictor using local area measurements (VIP++)," in Power Tech Proceedings, 2001 IEEE Porto, vol. 2. IEEE, 2001, pp. 6-pp.

[11] Y. Wang, I. R. Pordanjani, W. Li, W. Xu, T. Chen, E. Vaahedi, and J. Gurney, "Voltage stability monitoring based on the concept of coupled single-port circuit," IEEE Transactions on Power Systems, vol. 26, no. 4, pp. 21542163, Nov. 2011.

[12] M. Glavic, T. Van Cutsem, "A Short Survey of Methods for Voltage Instability Detection," Proceedings of 2011 IEEE PES GM, Detroit, MI, July 24-28, 2011.

[13] C. Bai, M. Begovic, R. Nuqui, D. Sobajic, Y. Song, "On Voltage Stability Monitoring with Voltage Instability Predictors," Proc. 2013 IEEE IREP Symposium, August 2013, Rethimno, Crete, Greece.

[14] M. Begovic, A. Peerzada, R. Nuqui, B. Picone, "On Networked VIP Monitoring of Voltage Stability, “ Proceedings 2017 IREP, Porto, Portugal, August 2017.

[15] M. Begovic and A. Phadke. "Control of voltage stability using sensitivity analysis", IEEE Trans. on Power Systems, Vol. 7, No 1, Feb. 1992, pp. 114-123.

[16] M. Begovic and A.G.Phadke: "Voltage Stability Assessment Through Measurement of the Reduced State Vector", paper 89 SM 709-7 PWRS, 1989 IEEE PES Summer Meeting, Long Beach, CA, July 1989. 